\title{
Reconfigurability and Reliability of Systolic/Wavefront Arrays
}

\author{
Edwin Hsing-Mean Sha and Kenneth Steiglitz, Fellow, IEEE
}

\begin{abstract}
In this paper, we study fault-tolerant redundant structures for maintaining reliable arrays. In particular, we assume the desired array (application graph) is embedded in a certain class of regular, bounded-degree graphs called dynamic graphs. We define the degree of reconfigurability $D R$, and $D R$ with distance $D R^{d}$, of a redundant graph. When $D R$ (respectively, $D R^{d}$ ) is independent of the size of the application graph, we say the graph is finitely reconfigurable, $F R$ (respectively, locally reconfigurable, $L R$ ). We show that $D R$ provides a natural lower bound on the time complexity of any distributed reconfiguration algorithm and that there is no difference between being $F R$ and $L R$ on dynamic graphs. We then show that if we wish to maintain both local reconfigurability and a fixed level of reliability, a dynamic graph must be of dimension at least one greater than the application graph. Thus, for example, a one-dimensional systolic array cannot be embedded in a one-dimensional dynamic graph without sacrificing either reliability or locality of reconfiguration.
\end{abstract}

Index Terms-Dynamic graphs, fault tolerance, reconfiguration, reliability, systolic arrays, wavefront arrays.

\section{INTRODUCTION}

$\mathbf{H}$ IGHLY PARALLEL pipelined structures such as systolic or wavefront arrays are attractive architectures for achieving high throughput [9]. Examples of important potential applications include digital signal processing [2], [11], large-scale scientific computation on arrays for solving partial differential equations [12], and simulating lattice-gas automata [14]. As such array processors become larger, the reliability of the processing elements (PE's) becomes a critical issue, and it is necessary to use fault tolerant techniques-both at the time of fabrication [15] and at run time. Defective PE's must be located, and the architecture reconfigured to substitute good PE's for bad.

In certain run-time applications, such as avionics and spaceflight, fault tolerant techniques must be able to restore proper operation as fast as possible after failures. For this purpose, distributed reconfiguration algorithms executed in parallel by the PE's themselves have been studied in [13] and [17]. In [5] a fault tolerant multiprocessor is developed for space applications that also employs a distributed reconfiguration approach for the topology of a chordal skip-link ring. In this paper, we study the complexity of algorithms for reconfiguring

Manuscript received September 10, 1990; revised January 15, 1992. This work was supported in part by National Science Foundation Grant MIP8912100 and U.S. Army Research Office-Durham Grant DDAL03-89-K-0074.

E. H.-M. Sha is with the Department of Computer Science and Engineering, University of Notre Dame, Notre Dame, IN 46556.

K. Steiglitz is with the Department of Computer Science, Princeton University, Princeton, NJ 08544.

IEEE Log Number 9208481. arrays after failures, and focus especially on run-time fault tolerance.

In most literature on fault tolerance, faults are confined to processing elements only, and it is assumed that all switches and connections [1], [3], [10], [18] are perfect. This is not valid when the number of switches and connections becomes large. In this paper we will use a graph model that takes into account failures of switches and interconnection wires as well as PE's. PE's and switches will be represented by nodes of the graph in the obvious way, and a connection between two elements in the computational structure will be represented by a node inserted in the edge between the appropriate two nodes in the graph model. Each node of the graph will have associated with it a probability of failure $\epsilon$.

To achieve fault tolerance, we add redundancy to the system. After a failure the original working architecture is reconfigured by replacing some nodes that were being used by redundant nodes. A good fault tolerant structure is one where the number of nodes that need to be changed after failure is as small as possible. In this paper, we define a measure of this adaptability, the degree of reconfigurability $(D R)$, and analyze this measure on a class of very regular graphs called dynamic graphs [6]-[8], [16]. We also analyze a stricter measure, called the degree of reconfigurability with distance, $D R^{d}$, which takes into account the total distance between original nodes and replacing nodes. Our goal is to investigate the relation between the structure of dynamic graphs, their reliability, and their fault tolerant capability as measured by their degree of reconfigurability.

The case when $D R$ is independent of the size of the system is especially important because it represents the situation when the amount of change necessary to repair the system depends only on the number of failed nodes, but not on the size of the system. In this case, we say the graph is finitely reconfigurable. Similarly, if $D R^{d}$, the total distance cost of changes is independent of the size of system, we say that it is locally reconfigurable.

Actually, in Section III, we show if the redundant system is a dynamic graph, it is locally reconfigurable if and only if it is finitely reconfigurable. Given a desired working structure, we will discuss what types of redundant structures are possible or impossible to maintain at a fixed level of reliability, while at the same time being locally reconfigurable. In particular, our main result is that, if we wish to maintain both local reconfigurability and a fixed level of reliability, the dynamic graph must be of dimension at least one greater than the application graph, which is shown in Sections IV and V. 


\section{Definitions AND Mathematical Framework}

A VLSI/wafer-scale-integration array architecture can be represented as a graph $G=(V, E)$. Each node of the graph $G$ can be regarded as a processor, and an edge of $G$ is a connection between two processors. We assume that the nodes fail independently, each with probability $\epsilon$. As mentioned earlier, a node in our graph model can represent a PE, a switch, or interprocessor connection.

Real working architectures are considered to be a family of graphs, $\mathcal{G}_{a}$, called application graphs; $G_{a}^{i}=\left(V_{a}^{i}, E_{a}^{i}\right)$ denotes the $i$ th application graph of $\mathcal{G}_{a}$. For example, $\mathcal{G}_{a}$ can be a family of linear arrays indexed by a number of nodes, so $G_{a}^{n}$ is an $n$-node linear array. We always assume each $G_{a}^{i}$ is connected and that for each value of $n$, there exists a unique $i$. Since we need to add redundant nodes or edges to increase reliability, the embedding structures, $\mathcal{G}_{r}$, called redundant graphs, are also represented as a family of graphs; $G_{r}^{i}=\left(V_{r}^{i}, E_{r}^{i}\right)$ denotes the $i$ th redundant graph of $\mathcal{G}_{r}$. Each pair of nodes in $V_{r}^{i}$ is associated with a value, distance, defined by a function $D^{i}: V_{r}^{i} \times V_{r}^{i} \rightarrow N$, where $N$ is the set of natural numbers; $D^{i}(a, a)=0$. This distance can be regarded as the physical distance between two nodes, or some cost, such as the communication cost.

Given two graphs $G_{1}=\left(V_{1}, E_{1}\right)$ and $G_{2}=\left(V_{2}, E_{2}\right)$, define the embedding function $\mu: V_{1} \rightarrow V_{2}$ such that $\left(v_{i}, v_{j}\right) \in E_{1}$ iff $\left(\mu\left(v_{i}\right), \mu\left(v_{j}\right)\right) \in E_{2}$. Let $\mu\left(V_{1}\right)$ be the image of $V_{1}$. Given an embedding function $\mu: V_{1} \rightarrow V_{2}$, let the mapping set $S(\mu)$ be the set of pairs, $\left\{(v, \mu(v)) \mid v \in V_{1}\right\}$. Thus, $S(\mu)-S\left(\mu^{\prime}\right)$ represents the difference between two embedding functions $\mu$ and $\mu^{\prime}$.

Given $\mathcal{G}_{a}$ and $\mathcal{G}_{r}$, the following function will determine which graph in $\mathcal{G}_{r}$ will be the redundant graph of the $i$ th application graph.

Definition 2.1: An embedding strategy for $\mathcal{G}_{a}$ and $\mathcal{G}_{r}$ is a function $E S: \mathcal{G}_{a} \rightarrow \mathcal{G}_{r}$, that is, if $\operatorname{ES}\left(G_{a}^{i}\right)=G_{r}^{j}, G_{r}^{j}$ is the redundant graph for $G_{a}^{i}$.

If $E S\left(G_{a}^{i}\right)=G_{r}^{j}$, and $k$ nodes of $G_{r}^{j}$ have failed, the failed nodes and all the edges incident to them will be removed and $G_{r}^{j}$ becomes a new subgraph $\hat{G}_{r}^{j}=\left(\hat{V}_{r}^{j}, \hat{E}_{r}^{j}\right)$. The procedure of finding a new embedding function $\mu_{k}^{i}: V_{a}^{i} \rightarrow \hat{V}_{r}^{j}$ is called reconfiguration.

Definition 2.2: Given $\mathcal{G}_{a}, \mathcal{G}_{r}$, and $E S$, the maximum fault tolerance of $G_{a}^{i}, \operatorname{MFT}\left(G_{a}^{i}\right)$, is the maximum number of nodes that can be allowed to fail arbitrarily in $\operatorname{ES}\left(G_{a}^{i}\right)$ such that $E S\left(G_{a}^{i}\right)$ can still find a subgraph isomorphic to $G_{a}^{i}$. In addition, $F T\left(G_{a}^{i}\right)$ is given, which is some fixed number $\leq \operatorname{MFT}\left(G_{a}^{i}\right)$ for each $i$.

Definition 2.3: Given $\mathcal{G}_{a}, \mathcal{G}_{r}, E S$, and fault tolerance $F T\left(G_{a}^{i}\right) \leq \operatorname{MFT}\left(G_{a}^{i}\right)$ for each $i$, the quadruple $\left(\mathcal{G}_{a}, \mathcal{G}_{r}, E S\right.$, $F T)$ is called an embedding architecture, $E A$.

For example, in Fig. 1, $\mathcal{G}_{a}$ is a family of linear arrays, and $\mathcal{G}_{r}$ is a family of triple-modular-redundancy (TMR) arrays obtained by triplicating each node of a linear array to be three nodes, called a module. Let $G_{r}^{n}=E S\left(G_{a}^{n}\right)$ be the $n$-module array, and let its corresponding $F T\left(G_{a}^{n}\right)$ be 2 for all $n$.

For simplicity, if the context is clear, we will always assume the $i$ th application graph maps to the $i$ th redundant graph, that

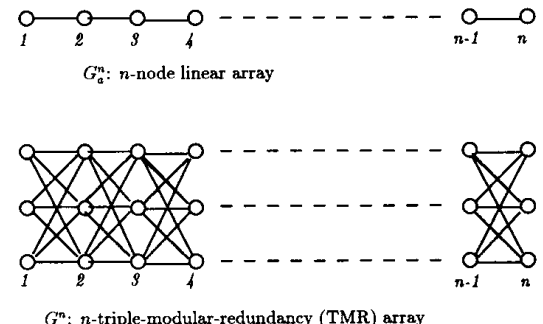

Fig. 1. Example of $\mathcal{G}_{a}$ and $\mathcal{G}_{r}$.

is, $E S\left(G_{a}^{i}\right)=G_{r}^{i}$. Let $\mu_{0}^{i}: G_{a}^{i} \rightarrow G_{r}^{i}$ be the initial embedding function for the $i$ th application graph $G_{a}^{i}$.

Definition 2.4: Given an embedding architecture, define the initial embedding, $I E$, to be a set of $\mu_{0}^{i}$ for all $G_{a}^{i}$ in the family.

For the above example in Fig. 1, an initial embedding can be a set of $\mu_{0}^{i}$ such that each node of $G_{a}^{i}$ maps to the bottom node of each module of $G_{r}^{i}$.

Given an embedding architecture for a $G_{a}^{i}$, after $k$ nodes have failed, obviously there may be many different embedding functions $\mu_{k}$ 's. However, the difference between $S\left(\mu_{0}^{i}\right)$ and $S\left(\mu_{k}^{i}\right)$ should be as small as possible for the purpose of real-time fault tolerance.

Suppose that the number of nodes in $G_{a}^{i}$ is $n$. Given $E A, I E$, and that $k \leq F T\left(G_{a}^{i}\right)$ nodes have failed, let the cost of reconfiguration of $G_{a}^{i}, \Delta(k, n)$, be the minimum of $\left|S\left(\mu_{0}^{i}\right)-S\left(\mu_{k}^{i}\right)\right|$ over all the possible embedding functions $\mu_{k}^{i}$, that is,

$$
\Delta(k, n)=\min _{\mu_{k}^{i}}\left|S\left(\mu_{0}^{i}\right)-S\left(\mu_{k}^{i}\right)\right|
$$

When there is no $\mu_{k}^{i}, \Delta(k, n)=\infty$. We also want to measure the total distance between original nodes and replacing nodes after reconfiguration. The total distance cost of reconfiguration for $G_{a}^{i}, \Delta^{d}(k, n)$, is similarly defined to be the following:

$$
\Delta^{d}(k, n)=\min _{\mu_{k}^{i}} \sum_{(a, b) \in S\left(\mu_{0}^{i}\right)-S\left(\mu_{k}^{i}\right)} D^{i}\left(\mu_{k}^{i}(a), b\right) .
$$

When there is no $\mu_{k}^{i}, \Delta^{d}(k, n)=\infty$. Under a given $E A$ and $I E$, let $D R(k, n)$, the degree of reconfigurability for $G_{a}^{i}$, be the maximum of $\Delta(k, n)$ over all possible $k$ failures in $G_{r}^{i}, k \leq F T\left(G_{a}^{i}\right)$, that is

$$
D R(k, n)=\max _{\substack{\text { failures of } k \text { nodes } \\ k \leq F T\left(G_{a}^{i}\right)}} \Delta(k, n) .
$$

The degree of reconfigurability with distance, $D R^{d}(k, n)$, is defined similarly (change $\Delta$ to be $\Delta^{d}$ in the preceding equation).

Return to the example in Fig. 1. Let the distance between two nodes in the same module be one, and the distance between two nodes, one in module $i$ and the other in module $j$, be $|i-j|+1$. In this case $D R(k, n)$ and $D R^{d}(k, n)$ for $G_{a}^{n}$ are both $k$, since for any $k \leq F T\left(G_{a}^{n}\right)=2$ faults, we need only change $k$ nodes in the same modules as the $k$ faulty nodes, and the distance between two nodes in the same module is one. 


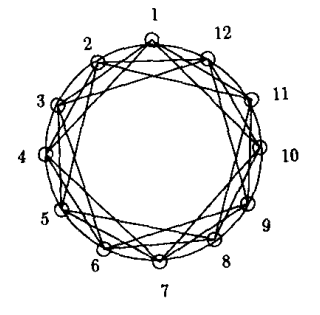

Fig. 2. Hayes' 4-FT single loop.

Definition 2.5: An embedding architecture, $E A$, is finitely reconfigurable (resp. locally reconfigurable), if there exists an initial embedding, $I E$, such that for all the $G_{a}^{i} \in \mathcal{G}_{a}, D R(k, n)$ [resp. $D R^{d}(k, n)$ ], can be bounded from above by a function of $k$ but not $n$.

For example, the embedding architecture for linear arrays in the preceding example is both $L R$ and $F R$, since for each $G_{a}^{i}, D R(k, n)=D R^{d}(k, n) \leq k$.

We show in the following lemma that Hayes' $h-F T(n+h)$ node single loop [4], which is an $h$-fault-tolerant graph for an $n$-node loop application graph, is not finitely reconfigurable.

The $n$th application graph $G_{a}^{n}$ is an $n$-node single loop, and the embedding strategy is to map $G_{a}^{i}$ to its so-called Hayes' $h$-FT $(n+h)$-node single loop. Thus, $G_{r}^{n}$ is defined by the following procedure, where we assume for this example that $h$ is even.

1. Form a single-loop graph $C_{n+h}$ with $n+h$ nodes.

2. Join every node $x_{i}$ of $C_{n+h}$ to all nodes at index distance $j$ from $x_{i}$, for all $j$ satisfying $2 \leq j \leq(h / 2)+1$.

The resulting graph $G_{r}^{n}$ is an $h-F T(n+h)$-node singleloop graph. Hayes [4] shows that is $\operatorname{MFT}\left(G_{a}^{n}\right)=h$. Let the distance between node $x_{i}$ and $x_{j}$ be $|i-j| \bmod n+h$. All the computations in the proof are based on indices $\bmod n+h$, and all the indices are in $G_{r}$. The graph in Fig. 2 is an example for $n=8, n=4$.

Lemma 2.1: The preceding embedding architecture with $F T=M F T=h$, mapping the $n$-node single loop to Hayes' $h$-FT $(n+h)$-node single-loop graph, is neither $F R$ nor $L R$ if $h$ is $O\left(n^{1 / 2}\right)$.

Proof: We assume there is an adversary $A$ who always tries best to select failures that show that $D R(k, n)$ is not bounded by a function of $k$ only. No matter what the initial $\mu_{0}^{n}$ is, $n$ working nodes must be distributed among the $n+h$ nodes of $G_{r}^{n}$. Define a segment $S$ to be a sequence of consecutively numbered working nodes $\left(x_{i}, x_{i+1}, \cdots, x_{j}\right)$ in $G_{r}^{n}$, where $x_{i-1}$ and $x_{j+1}$ are nonworking redundant nodes. Denote the length of the segment $S$ by $l(S)=j-i+1$, and suppose the $h$ nonworking nodes, ordered by their indices, form the sequence $\left(x_{i_{1}}, x_{i_{2}}, \cdots, x_{i_{h}}\right)$. For each $x_{i_{j}}$ there is a segment $S_{j}$ (it may be null) starting from $x_{i_{j}+1}$. Thus,

$$
\sum_{j=1}^{n}\left(l\left(S_{j}\right)+1\right)=n+h .
$$

There must exist a segment $S^{*}$ such that $l\left(S^{*}\right)+1 \geq(n+h) / h$; that is, $l\left(S^{*}\right) \geq n / h$. Without loss of generality, assume that $S^{*}$ is from node $x_{1}$ to node $x_{l\left(S^{*}\right)}$.
The adversary can choose the middle node $x_{d}$ of segment $S^{*}$ to be faulty, that is, $d=\lceil n / 2 h\rceil$. Choose a reconfiguration that is optimal in the sense that the fewest possible number of nodes in $G_{r}^{n+h}$ are changed. Let $m$ be the number of nodes in $S^{*}$ which are changed in this reconfiguration. Let $C$ be such a sequence of $m$ nodes $\left(x_{j_{1}}, x_{j_{2}}, \cdots, x_{j_{m}}\right)$ ordered by their indices. We know $x_{d}$ must be replaced by one node, say $x_{d}^{\prime}$, and if $x_{d}^{\prime}$ is a working node, it must be replaced by another node. Thus, there is a sequence $\subseteq C$ of working nodes in $S^{*}$ in this sequence of replacements, starting with $x_{d}$ and ending at a working node that is replaced by the first node $x_{r}$ outside $S^{*}$. First, we divide $S^{*}$ into many small subsegments with length $w$, where $w=2[(h / 2)+1]$, and represent them as a sequence $\left(S_{1}^{*}, \cdots, S_{k}^{*}\right)$. Let $x_{d}$ be in subsegment $S_{i}^{*}$. Without loss of generality, assume that the index of $x_{r}$ is larger than the largest index of a node in $C$; that is, $r>j_{m}$.

We claim that there must exist at least one node in $C$ in the subsegment $S_{k}^{*}$ or $S_{1}^{*}$. Suppose not. Let $x_{r}$ replace $x_{i}$ in $C$ and let $a$ and $b$ be the two nodes connected to $x_{i}$ in the initial working subgraph. Since connections must be of length at most $(h / 2)+1$ and the distance between $x_{i}$ and the last node in $S^{*}$ (and also the first node in $S^{*}$ ) is $>w$, we know $a$ and $b$ must be in $S^{*}$. If $a$ or $b$ is not in $C$, say $a$, because $a$ is not replaced, $x_{r}$ must be connected to $a$ after the reconfiguration. But we know that $i \leq j_{m}$ and $r>l\left(S^{*}\right)$ from the assumption, so it is impossible that $x_{r}$ is connected to $a$. Thus, we know that $a$ and $b$ are in $C$, say that $a$ is replaced by $a^{\prime}$. Denote the sequence of original working nodes starting from $x_{i}$ toward one direction in the original working subgraph by $\left\{x_{i}, a, a_{1}, a_{2}, \cdots\right\}$, and the sequence after reconfiguration by $\left\{x_{r}, a^{\prime}, a_{1}^{\prime}, a_{2}^{\prime}, \cdots\right\}$. If $a^{\prime} \in S^{*}$, because $a^{\prime}$ replaces $a, a^{\prime}$ must be in $C$. Since the index of $a^{\prime}$ is $\leq j_{m}$, it is impossible for $a^{\prime}$ to be connected to $x_{r}$. Thus, $a^{\prime}$ is not in $S^{*}$. In summary, we know that if $x_{i} \in C$ and $x_{r} \notin S^{*}$, then $a$ is in $C$ and $a^{\prime}$ is not in $S^{*}$. Repeating the argument, using $a$ instead of $x_{i}$ and $a^{\prime}$ instead of $x_{r}$, we can get the result that $a_{1}$ is in $C$ and $a_{1}^{\prime}$ is not in $S^{*}$. Continuing in this way, it follows that all the nodes $a, a_{1}, a_{2}, \cdots$ are in $C$ and nodes $a^{\prime}, a_{1}^{\prime}, a_{2}^{\prime}, \cdots$ are not in $S^{*}$, but this is impossible, since there are only a finite number of nodes in $C$. Thus, our claim is correct.

We claim next that in each pair of the subsegments $\left(S_{l}^{*}, S_{k-l+1}^{*}\right)$, where $l=1, \cdots, i$, there exists at least one node in $C$. We have proved that it is true for the first pair of subsegments $\left(S_{1}^{*}, S_{k}^{*}\right)$. Assume it is true for all the pairs of subsegments from $l=1$ to $k-j$, and $i<j$. We represent $C^{\prime}=\left\{x_{j} \mid x_{j} \in C, x_{j}\right.$ not in $S_{1}^{*}, \cdots, S_{k-j}^{*}$, and $\left.S_{j+1}^{*}, \cdots, S_{k}^{*}\right\}$. Since $x_{d} \in C^{\prime}$, from the way that $x_{r}$ is chosen, we know there must exist one node in $C^{\prime}$ which is replaced by a node outside of $C^{\prime}$. If, in $S_{k-j+1}^{*}$ and $S_{j}^{*}$, there does not exist a node in $C^{\prime}$, the same argument as above results in the same contradiction. Thus, in each pair of subsegments in $S^{*}$, there is at least one node that has been replaced. The number of nodes in $C$ must therefore be at least $n / 2 h w=\Omega\left(n / h^{2}\right)$. If $h=o\left(n^{1 / 2}\right)$, a number of nodes that is an unbounded function of $n$ need to be changed. Thus, $D R(k, n)$ is not bounded by a function of $k$ only, under any initial embedding function $\mu_{0}^{n}$, and therefore the Hayes' embedding architecture is not finitely reconfigurable. It is obvious that the total distance between 

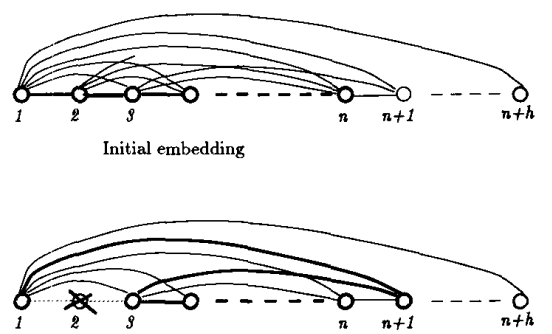

After reconfiguration

$G_{+}^{\text {n: }}:(n+h)$-node complete graph

Fig. 3. Example that is $F R$ but not $L R$.

original nodes and their replacing nodes is also an increasing function of $n$, so it is not $L R$ either.

Our next example is an embedding architecture that is finitely reconfigurable, but not locally reconfigurable. Choose $\mathcal{G}_{a}$ as in Fig. 1 to be a family of linear arrays, and $\mathcal{G}_{r}$ as in Fig. 3 to be a family of complete graphs on a row. Let $E S$ map $G_{a}^{n}$ to $G_{r}^{n+h}$ and let $F T\left(G_{a}^{n}\right)=h$, for each $G_{a}^{n}$ in $\mathcal{G}_{a}$. The distance between node $i$ and node $j$ is defined to be $|i-j|$. After one node has failed, say node 2 , we can take any spare node to replace it, say node $n+1$, as shown in Fig. 3 .

Lemma 2.2: If $h$ is $O(n)$, the preceding embedding architecture is $F R$, but not $L R$.

Proof: It is obvious that such an $E A$ is finitely reconfigurable, since any spare node can replace any other node, so that only $k$ faulty nodes need to be changed after $k$ nodes fail. Considering $G_{a}^{n}$ and $G_{r}^{n+h}$, under any initial embedding, there must exist a sequence of working nodes in $G_{r}^{n+h}$ with consecutive indices of length $\geq n /(h+1)$, by the same argument as in Lemma 2.1. Choosing the middle node of such a path to be faulty, the distance between any spare node and the faulty node must be $\geq n /(2(h+1))$. Since $h=O(n)$, the distance is an increasing function of $n$. Thus, this $E A$ is not locally reconfigurable.

\section{Degree of ReConfigurability FOR DyNAMIC GRAPHS}

In applications we are interested in graphs that are very regular and of bounded degree. An interesting and useful class of such graphs are called dynamic graphs [6]-[8], [16], which model regular systolic and wavefront arrays in a natural way. An undirected $k$-dimensional dynamic graph $G^{k}=$ $\left(V^{k}, E^{k}, T^{k}\right)$ is defined by a finite digraph $G^{0}=\left(V^{0}, E^{0}\right)$, called the static graph, and a $k$-dimensional labeling of edges $T^{k}: E^{0} \rightarrow Z^{k}$. The vertex set $V_{x}$ is a copy of $V^{0}$ at the integer lattice point $x$ and $V^{k}$ is the union of all $V_{x}$, where $x \in Z^{k}$. Let $a_{x}$ be the copy of node $a \in V^{0}$ in the vertex set $V_{x}$ and let $b_{y}$ be the copy of node $b \in V^{0}$ in the vertex set $V_{y}$. Nodes $a_{x}$ and $b_{y}$ are connected if $(a, b) \in E^{0}$, and the difference between the two lattice points $y$ and $x$ is equal to the labeling $T^{k}(a, b)$. Therefore, the dynamic graph is a locally finite, infinite graph consisting of repetitions of the basic cell $V^{0}$ interconnected by edges determined by the labeling $T^{k}$. In Fig. 4, we show an example of a 2-D static graph $G^{0}$ and its corresponding dynamic graph $G^{2}$.
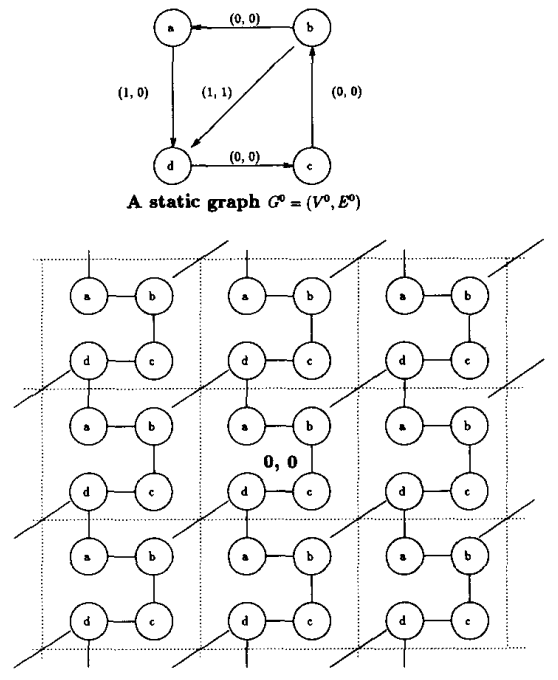

Fig. 4. Example of $G^{0}$ and the corresponding dynamic graph $G^{2}$.

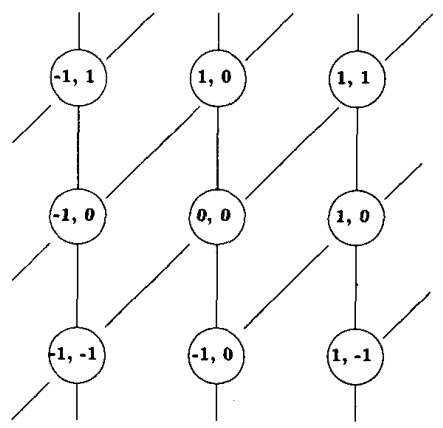

Fig. 5. Cell-dynamic graph $G_{c}$ of $G^{2}$.

For $x, y \in Z^{k}$, let $E_{x, y}=\left\{\left(a_{x}, b_{y}\right) \mid(a, b) \in E^{0}\right\}$. The graph with vertex set $V_{x}$ and edges with both endpoints only in $V_{x}$ is called the $x$ th cell of $G^{k}, C_{x}=\left(V_{x}, E_{x, x}\right)$. Given a dynamic graph, we can contract all the nodes in the same cell to one node and delete the edges totally within the cell. This contracted graph is called the cell-dynamic graph, $G_{c}=\left(V_{c}, E_{c}\right)$, where $V_{c}=Z^{k}$ and $E^{c}=\cup_{x \neq y} E_{x, y}$. We give an example in Fig. 5 , which is the cell-dynamic graph corresponding to $G^{2}$ in Fig. 4.

Given a static graph $G^{0}$, we define $F_{j}$ to be the finite subgraph of $G^{k}$ such that each dimension of $F_{j}$ has $j$ cells, that is, $F_{j}=\left(\cup_{x} V_{x}, \cup_{x, y} E_{x, y}\right)$, where $x=\left(x_{1}, x_{2}, \cdots, x_{k}\right), 1 \leq$ $x_{i} \leq j$, and $y=\left(y_{1}, y_{2}, \cdots, y_{k}\right), 1 \leq y_{i} \leq j$. We define the family $\mathcal{F}$ of $k$-dimensional dynamic graphs to be the set of $F_{j}$, where $j \geq 1$.

There are different ways to define distance in dynamic graphs. For example, one resonable definition of the distance function $D$ is to define the distance between two nodes, one in vertex set $V_{x}$ and the other in $V_{y}$, to be the Euclidean distance in $k$-dimensional space between point $x$ and point $y$ if $x$ and $y$ are in different cells, and one if they are in the same cell. We say that a distance function $D$ satisfies property $\nabla$ (triangle 
inequality), if the distance between nodes $a$ and $b$ is less than or equal to the total distance of any path from $a$ to $b$. Of course, Euclidean distance satisfies $\nabla$. The following lemma will show that when the set of redundant graphs $\mathcal{G}_{r}$ is a family of dynamic graphs and the distance function satisfies $\nabla$, then any embedding architecture is $L R$ if and only if it is $F R$. In the rest of this paper, we assume that $D$ satisfies property $\nabla$.

Lemma 3.1: When $\mathcal{G}_{r}$ is a family of dynamic graphs and its distance function satisfies $\nabla$, the embedding architecture is locally reconfigurable if and only if it is finitely reconfigurable.

Proof: Given an $E A$, if this $E A$ is $L R$, we know by definition that the total distance cost of any $k$ failures can be expressed as a function $f(k)$, where $f$ is a function of $k$ only. We know the distance between any two nodes is at least one, so the number of nodes changed must be $\leq f(k)$. Thus, this $E A$ is also $F R$.

Suppose that it is $F R$. We know that for each $G_{a}^{n} \in \mathcal{G}_{a}$, after $k$ nodes have failed, at most a function of $k$, say, $f(k)$, nodes must be changed in the original working subgraph. Let $a_{1}$ be the node in $G_{a}^{n}$ such that the distance in $G_{r}^{n}$ between $\mu_{k}^{n}\left(a_{1}\right)$ and $\mu_{0}^{n}\left(a_{1}\right)$ is the maximum over all the nodes in $V_{a}^{n}$

Because there are at most $f(k)$ nodes that are changed by $\mu_{k}^{n}$, there exists a path in the application graph $G_{a}^{n}$ with at most $f(k)$ edges from $a_{1}$ to an unchanged node $a_{2}$, that is, $\mu_{0}^{n}\left(a_{2}\right)=\mu_{k}^{n}\left(a_{2}\right)$. Let $c$ be the maximum distance between any two nodes connected by an edge, which is a constant independent of $k$ and $n$ by definition. The distance $D$ between node $\mu_{0}^{n}\left(a_{1}\right)$ and $\mu_{0}^{n}\left(a_{2}\right)$ is at most $c \cdot f(k)$ by property $\nabla$, the triangle inequality. Similarly, the distance between node $\mu_{k}^{n}\left(a_{1}\right)$ and node $\mu_{k}^{n}\left(a_{2}\right)$ is at most $c \cdot f(k)$. Since $\mu_{k}^{n}\left(a_{2}\right)=\mu_{0}^{n}\left(a_{2}\right)$, the distance between $\mu_{0}^{n}\left(a_{1}\right)$ and $\mu_{k}^{n}\left(a_{1}\right)$ is at most $2 c \cdot f(k)$. Therefore the total distance of the $f(k)$ changed nodes is at most $2 c \cdot f(k)^{2}$ because there are at most $f(k)$ pairs that are changed. $E A$ is therefore locally reconfigurable from the definition.

Finite reconfigurability is desirable in practice, especially for real-time fault tolerance, because it shows that after $k$ nodes have failed, at most a function of $k$ nodes need to be changed, independent of the size of the application graph. Lemma 3.2 will show that the degree of reconfigurability $D R$ provides a lower bound on the time complexity of any distributed reconfiguration algorithm, and shows one reason this measure $D R$ is important. We assume in what follows that it takes one time step to send a message through an edge.

Lemma 3.2: When $G_{a}^{i}$ is an $n$-node application graph and $\mathcal{G}_{r}$ is a family of $d$-dimensional dynamic graphs, the time complexity of any distributed reconfiguration algorithm is $\Omega\left[(D R / k)^{1 / d}\right]$, where $k$ is the number of nodes that have failed.

Proof: After $k$ nodes have failed, we must change at least $D R$ nodes to reconfigure. We can assume that a distributed reconfiguration algorithm is initiated by a neighbor node, called a source node, of each faulty node after this neighbor node has detected the failure. We need to inform at least $D R$ nodes in $G_{r}^{i}$ that they are assigned different nodes in $G_{a}^{i}$. Thus, the time to broadcast this fault information is a lower bound on the time complexity of any distributed reconfiguration algorithm.
Let the corresponding static graph be $G^{0}=\left(V^{0}, E^{0}\right)$, and its labeling be $T^{d}$. The maximum edge distance $c$ in one dimension is the $\max \left\{\left|t_{i}\right| \mid\left(t_{1}, \cdots, t_{i}, \cdots, t_{d}\right) \in T^{d}(e), e \in\right.$ $\left.E^{0}\right\}$. Let $m$ be equal to $\left(\left|V^{0}\right| \times 2 c\right)^{d}$. We can always contract the nodes of $G^{d}$ into groups of at most $m$ nodes to obtain a $d$-dimensional reduced graph $G_{c}^{\prime}=\left(V_{c}^{\prime}, E_{c}^{\prime}\right)$, such that $V_{c}^{\prime}=Z^{d}$ and $E_{c}^{\prime}=\left\{(x, y) \mid x, y \in V_{c}^{\prime}, x \neq y, y-x=\right.$ $\left(e_{1}, \cdots, e_{i}, \cdots, e_{d}\right)$ where $e_{i}=0$ or 1$\}$. Each node of $V_{c}^{\prime}$, called a class here, represents at most $m$ nodes of the dynamic graph. Note that $m$ is a constant by definition.

After $t$ time steps, one source node can inform at most $(2 t)^{d}$ classes in a $d$-dimensional reduced graph, so at most $(2 t)^{d} m$ nodes have been reached. Since there are at most $c_{1} k$ source nodes, where $c_{1}$ is the maximum degree in $G_{r}$, the total number of nodes that can be informed after $t$ time steps is at most $(2 t)^{d} m k$. There are $D R$ nodes that need to be informed, so $t$ should be at least $\Omega\left[(D R / k)^{1 / d}\right]$.

\section{IMPOSSIBILITY OF AN $L R$-RELIABLE EMBEDDING OF DYNAMIC GRAPHS FROM DIMENSION $d$ TO $d$}

In this section, we restrict attention to dynamic graphs, and consider the relationship between reconfigurability and reliability. In particular, we ask whether a given embedding architecture can be finite and locally reconfigurable, and at the same time maintain a given level of reliability. Without the constraint of being $F R$ or $L R$, we can simply construct a redundant graph to be many replications of the application graph, achieving high reliability, but at the price of using large amounts of hardware and being difficult to reconfigure. Our main result is Theorem 4.5: when mapping from $d$ dimensions to $d$-dimensions, we cannot maintain both local reconfigurability and reliability simultaneously.

As Lemma 3.1 shows, there is no difference beween local and finite reconfigurability for dynamic graphs, and thus we consider only local reconfigurability, without loss of generality. We define $L R$ reliability in our framework as follows. Given an $E A$ which is $L R$, the probability, for each $i$, that $G_{r}^{i}$ contains an isomorphic image of $G_{a}^{i}$ is

$$
P\left(G_{a}^{i}\right)=\sum_{k=0}^{F T} \epsilon^{k}(1-\epsilon)^{n-k}\left(\begin{array}{l}
n \\
k
\end{array}\right)
$$

where $n=\left|V_{r}^{i}\right|$. The following definition replaces Definition 2.5 in the statistical case.

Definition 4.1: An embedding architecture is $L R$ reliable with reliability $\beta$, if $P\left(G_{a}^{i}\right) \geq \beta$ for all the $G_{a}^{i} \in \mathcal{G}_{a}$.

The following lemma is useful in what follows.

Lemma 4.1: Given $\mathcal{G}_{a}, \mathcal{G}_{r}$, and $E S$, for each $i$, let $\operatorname{MFT}\left(G_{a}^{i}\right)$ be the maximum number of failures that allows the corresponding $E A$ to be $L R$. If this $M F T$ is upper-bounded by a constant as $n \rightarrow \infty$, there exists a constant $\beta$ such that $E A$ cannot be $L R$ reliable with reliability $\beta$.

Proof: Let the upper bound on $M F T$ be $c$. By the definition of $M F T$ in the hypothesis of the lemma, there exist $c+1$ nodes in the redundant graph $G_{r}^{i}$ such that after they have 
failed, for any $I E, E A$ cannot be $L R$.

$$
P\left(G_{a}^{i}\right)<\sum_{k=0}^{c+1} \epsilon^{k}(1-\epsilon)^{n-k}\left(\begin{array}{l}
n \\
k
\end{array}\right) .
$$

We know $n$ can be chosen large enough to make $c+1<\epsilon n$, so the term corresponding to $k=c+1$ is the largest in the summation. Thus, the probability $P\left(G_{a}^{i}\right)<(c+1)(1-$ $\epsilon)^{n-c-1}\left(\begin{array}{c}n \\ c+1\end{array}\right)$. Since $(1-\epsilon)^{n-c-1} \leq e^{-\epsilon(n-c-1)}$, and $\left(\begin{array}{c}n \\ c+1\end{array}\right) \leq n^{c+1} /(c+1)$ !, it is obvious that when $n$ goes to $\infty, P\left(G_{a}^{i}\right)$ goes to 0 . Thus, for some $i$, we always can pick a $\beta>P\left(G_{a}^{i}\right)$. Therefore, such an embedding architecture cannot be $L R$ reliable with reliability $\beta$.

We want to study some properties of dynamic graphs if we insist on local reconfigurability after some nodes have failed, since local reconfigurability is desirable in practical implementations. The following lemma tells us that onedimensional dynamic graphs cannot be $L R$ reliable when the application graphs are linear arrays.

Lemma 4.2: When $\mathcal{G}_{a}$ is a family of one-dimensional linear arrays and $\mathcal{G}_{r}$ is a family of one-dimensional dynamic graphs, there exists a constant $\beta$ such that no embedding architecture is $L R$ reliable with reliability $\beta$.

Proof: As in the proof of Lemma 3.2, we can always build a reduced graph $G_{c}^{\prime}=\left(V_{c}^{\prime}, E_{c}^{\prime}\right)$ by contracting sets of size at most $m$ nodes in $G_{r}^{n}$ to produce a one-dimensional linear array. Each node of $G_{c}^{\prime}$ now represents a class of a finite number of nodes. Note that $m$ is a constant number, since $G^{0}$ is a finite graph by definition.

For any initial embedding, the $n$ nodes of $G_{a}^{n}$ are distributed into at least $n / m$ contiguous classes in $G_{c}^{\prime}$. If the adversary chooses all the nodes in the middle class of the preceding $n / m$ classes to be faulty, the initial working subgraph is separated into two halves. We must shift at least half of the $G_{a}^{n}$ and, therefore, change $\Omega(n)$ nodes to get a new working subgraph. Thus, if an embedding architecture is locally reconfigurable, its $F T$ must be bounded by a constant $m$. From Lemma 4.1, we know there exists a constant $\beta$, such that $E A$ cannot be $L R$ reliable with reliability $\beta$.

To generalize Lemma 4.2 , we define an $n^{d}$-node $d$ dimensional web to be a $d$-dimensional graph $G_{l}=\left(V_{l}, E_{l}\right)$ such that $V_{l}=\left\{x=\left(x_{1}, x_{2}, \cdots, x_{d}\right) \mid\right.$ where $x_{i}=$ $0, \cdots, n-1\}$ and $E_{l}=\left\{(x, y) \mid x, y \in V_{l}, x \neq y, y-x=\right.$ $\left(e_{1}, \cdots, e_{i}, \cdots, e_{d}\right)$ where $e_{i}=0$ or 1$\}$. Thus, we connect all adjacent points in the $d$-dimensional Euclidean space. For example, Fig. 6 shows a 2-D 16-node web. The family of $d$-dimensional webs is indexed by $n$.

Theorem 4.3: If $\mathcal{G}_{a}$ is a family of $d$-dimensional webs and $\mathcal{G}_{r}$ is a family of $d$-dimensional dynamic graphs, there exists a constant $\beta$ such that no embedding architecture is $L R$ reliable with reliability $\beta$.

Proof: We can always find a $d$-dimensional reduced graph $G_{c}^{\prime}=\left(V_{c}^{\prime}, E_{c}^{\prime}\right)$ by contracting the dynamic graph $G_{r}^{n}$ as we did in the proof of Lemma 3.2. Without loss of generality, we consider the most general case with all possible edges present, where $V_{c}^{\prime} \subset Z^{d}$ and $E_{c}^{\prime}=\left\{(x, y) \mid x, y \in V_{c}^{\prime}, x \neq\right.$

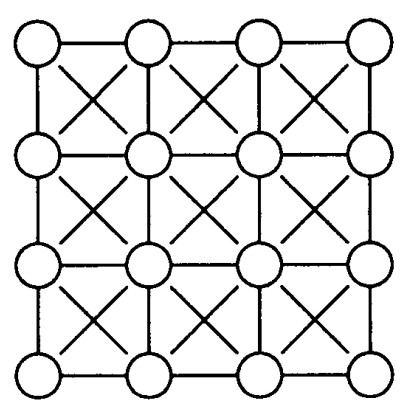

Fig. 6. Example of a 2-D 16-node web.

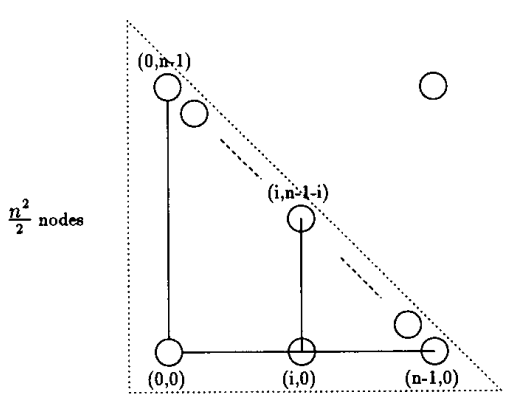

Fig. 7. $n$ paths in the proof of Theorem 4.3.

$y, y-x=\left(e_{1}, \cdots, e_{i}, \cdots, e_{d}\right)$ where $e_{i}=0$ or 1$\}$. Each node of $V_{c}^{\prime}$ represents a class of $m$ modes of $G_{r}^{n}$, where $m$ is the constant in the proof of Lemma 3.2.

First, we prove that there cannot be an embedding strategy that maps a $d$-dimensional web to a $(d-1)$-dimensional dynamic graph. Suppose first an $n \times n$ 2-D lattice is projected to a one-dimensional dynamic graph. Among the $n^{2}$ nodes in the web, the vertices on the path from vertex $(0,0)$ to $(0, n-1)$ must be projected to at most $n$ consecutive classes. Similarly, each of the $n$ paths horizontally from $(0,0)$ through $(i, 0)$ and vertically to the diagonal vertices $(i, n-1-i)$ where $0 \leq i \leq n-1$ also must be projected to at most $n$ consecutive classes. We show these $n$ paths in Fig. 7. Thus, all the $n^{2} / 2$ nodes on the paths must be in at most $2 n$ classes, and there must exist one class to which at least $n / 4$ nodes are mapped. This is impossible, since each class only has a finite number of nodes. The same argument can be generalized easily to $d$-dimensional lattices. Thus, we can restrict attention to the possibility of mapping a $d$-dimensional web to a $d$-dimensional dynamic graph.

We say a class in $G_{c}^{\prime}$ is empty if there is no working node in it. In the application graph the nodes that are adjacent must be mapped to one or adjacent classes. It is not difficult to see that in the initial embedding there cannot be an empty class surrounded by nonempty classes. Consider a line of $\geq n$ nodes in the $n^{d}$-node $d$-dimensional web, as in the proof of Lemma 4.2. For any initial embedding these $n$ nodes are distributed into at least $n / m$ classes that are linearly connected in $G_{c}^{\prime}$. These images of lines may zig-zag in $G_{c}^{\prime}$, but must map to at least $n / m$ contiguous classes. Therefore, there is a welldefined inner central class which is $\Omega(n / m)$ classes away 


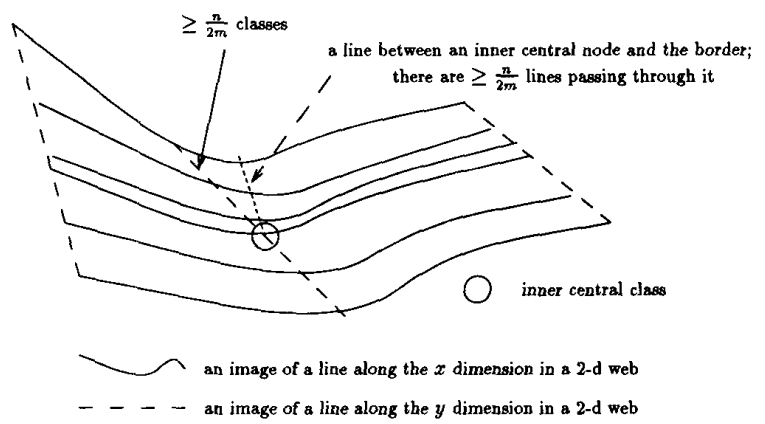

Fig. 8. Inner central class in the proof of Theorem 4.3 .

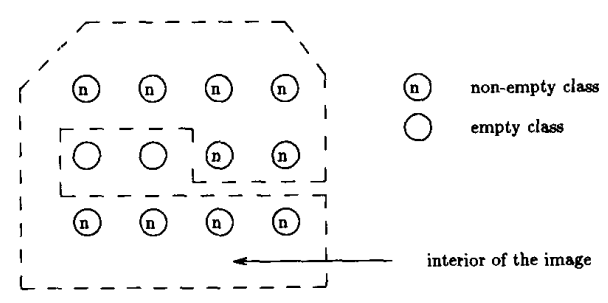

Fig. 9. Pseudohole.

from the border in the image of the web, as shown in Fig. 8 . Note that a line between the inner central class and the border may not be the image of a line along one dimension in the web, but the line must contain $\Omega(n)$ nodes in the web, as Fig. 8 shows.

If the adversary chooses all the nodes, at most $m$, in the inner central class to be faulty, the original working subgraph has a central inner hole. We must change $\Omega(n)$ nodes in one direction to get a new isomorphic subgraph in $G_{r}^{n}$. Therefore, to maintain local reconfigurability, for any embedding architecture, FT must be upper-bounded by $m$. From Lemma 4.1, we then know there exists a constant $\beta$, such that $E A$ cannot be $L R$ reliable with reliability $\beta$.

We next modify the application graph so that each node $x=$ $\left(x_{1}, x_{2}, \cdots, x_{d}\right)$ is connected only to nodes $y=\left(x_{1}, \cdots, x_{i} \pm\right.$ $\left.1, \cdots, x_{d}\right), i=1, \cdots, d$. We call such a $d$-dimensional graph a $d$-dimensional orthogonal lattice. To develop intuition for the general case of $d$-dimensional dynamic graphs, the following lemma extends Theorem 4.3 to 2-D orthogonal lattices.

Lemma 4.4: If $\mathcal{G}_{a}$ is a family of 2-D orthogonal lattices and $\mathcal{G}_{r}$ is a family of 2-D dynamic graphs, there exists a constant $\beta$ such that no embedding architecture is $L R$ reliable with reliability $\beta$.

Proof: As in the proof of Theorem 4.3, we know that a 2-D orthogonal lattice cannot be embedded in a onedimensional dynamic graph (we made no use of diagonal edges in that proof). Without diagonal edges, however, the rest of the proof is a bit more complicated.

An image of an application graph can be regarded as a polygon. We say an embedding in $G_{c}^{\prime}$ has a hole of size $k$, if there exist $k$ consecutive empty classes in a line along one dimension which are inside the polygon and surrounded by nonempty classes. Thus, the example in Fig. 9 is excluded from our definition of hole.

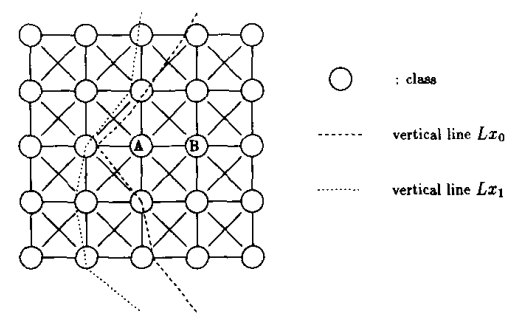

Fig. 10. Image of vertical lines $L x_{0}$ and $L x_{1}$.

We claim that after any embedding of a 2-D orthogonal lattice in a 2-D dynamic graph, it is impossible that there is a hole of size 2 . Assume our claim is false, and denote the empty classes in a hole of size 2 by $A$ and $B$. Index the nodes in the 2-D orthgonal lattice $G_{a}$ by $x_{i j}$. For notational convenience, choose the origin so that $x_{00}$ is a particular node which is mapped to the nonempty class immediately above $A$ in $G_{c}^{\prime}$. We will refer to the vertical line in $G_{a}$ passing through $x_{i j}$ as the vertical line $L x_{i}$.

We have the following observations about the images in $G_{c}^{\prime}$ of vertical lines in the orthogonal lattice $G_{a}$. First, the images of the vertical lines $L x_{i}$ and $L x_{i+1}$ cannot be more than one class apart along one dimension. Because the image of each pair of nodes $x_{i j}$ and $x_{i+1, j}$ is in the same class or adjacent classes, this follows by induction on $j$. Second, the vertical lines $L x_{0}$ and $L x_{1}$ (respectively, $L x_{0}$ and $L x_{-1}$ ) must pass on the same side of $A$ and $B$, as in Fig. 10, since there is no edge passing between $A$ and $B$. According to the preceding two observations, by induction on $i$, all the vertical lines $L x_{i}$ must be on the same side of $A$ and $B$ (either left or right), so $A$ and $B$ cannot be in the interior of the image of $G_{a}$. This contradiction proves that it is impossible to have a hole of size 2 . As we did in Theorem 4.3, the adversary can choose the two inner central classes in one dimension to be faulty, and, as before, there is no way to reconfigure $G_{r}$ so that those two faulty classes are surrounded by nonempty classes. Thus, we must change $\Omega(n)$ nodes in one dimension to get a new working subgraph.

Finally, we can extend this result to $d$ dimensions. The line containing classes $A$ and $B$ will be replaced by a $(d-1)$ dimensional hyperplane in a $d$-dimensional dynamic graph.

Theorem 4.5: If $\mathcal{G}_{a}$ and $\mathcal{G}_{r}$ are families of $d$-dimensional dynamic graphs, there exists a constant $\beta$ such that no embedding architecture can be $L R$ reliable with reliability $\beta$.

Proof: Given an application graph $G_{a}$, which is a dynamic graph, a reduced graph can be built as before. Since the application graph is connected and a class is connected only to its neighboring classes, there exists at least one edge along each dimension from one class to its neighboring class. Therefore, any $d$-dimensional reduced graph contains a subgraph that is isomorphic to a $d$-dimensional orthogonal lattice. We, therefore, need only prove the theorem for the case of the application graph being a family of $d$-dimensional orthogonal lattices. Again, the proof of Theorem 4.3 shows that $d$-dimensional orthogonal lattices cannot be embedded in $(d-1)$-dimensional dynamic graphs. 


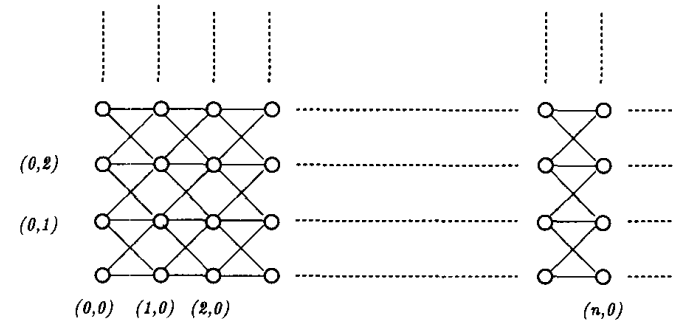

Fig. 11. LR-reliable 2-D dynamic graph.

We claim that it is impossible that there exist a hole of size $2^{d-1}$ in one hyperplane $H$ along $(d-1)$ dimensions (one coordinate is fixed) in the reduced graph. Assume our claim is false. Call the preceding $2^{d-1}$ classes an obstacle $O$. The obstacle is composed of two empty classes along each of the $(d-1)$ dimensions in $H$. Call the fixed dimension of $H$ "vertical." By the same reasoning as in Lemma 4.4 , no vertical lines can pass through the obstacle $O$, and the images of any two adjacent vertical lines must lie on the same side of the obstacle $O$ in the reduced graph. Therefore, the obstacle cannot be in the interior of the reduced graph, so our claim is correct. The adversary then chooses the inner central $2^{d-1}$ classes in $H$ to be faulty. There is no way to reconfigure the redundant graph such that those faulty classes are surrounded by nonempty classes. Thus, we must change $\Omega(n)$ nodes in one dimension to get a new isomorphic subgraph.

\section{POSSIBILITY OF AN $L R$-RELIABLE EMBEDDING OF DYNAMIC GRAPHS FROM DIMENSION $d$ TO $d+1$}

Finally, we want to show that we really can embed $d$ dimensional dynamic graphs in $(d+1)$-dimensional dynamic graphs, while maintaining any desired high reliability and local reconfigurability. We begin with the one-dimensional case.

Lemma 5.1: When $\mathcal{G}_{a}$ is a family of linear arrays, there exists an embedding architecture where $\mathcal{G}_{r}$ is a family of 2-D dynamic graphs, which can be $L R$ reliable with any given $\beta$.

Proof: We prove this by constructing a redundant graph $G_{r}^{n}$ for an $n$-node linear array $G_{a}^{n}$ as shown in Fig. 11. $G_{r}^{n}$ has $n$ columns and each column has $s$ nodes. Let $F T\left(G_{a}^{n}\right)<s$.

The initial embedding allocates each node of $G_{a}^{n}$ to a distinct column of $G_{r}^{n}$, that is, let the initial isomorphic subgraph be the sequence $(0,0),(1,0), \cdots,(n, 0)$. If one node $(i, 0)$ has failed, we choose $(i, 1)$ as the replacing node, and if nodes $(i, 0)$ and $(i, 1)$ have failed, we use $(i-1,1),(i, 2)$, and $(i+1,1)$ to replace nodes $(i-1,0),(i, 0)$, and $(i+1,0)$. By using the preceding reconfiguration procedure, we change at most $2 k-1$ nodes after any $k<s$ nodes have failed. Since $D R(k, n)=O(k), G_{a}^{n}$ with respect to such an $E A$ and $I E$ is locally reconfigurable.

We now want to show that given $\beta$, we can find an $s$ and $G_{r}^{n}$ with the desired properties. Let $\hat{G}_{r}^{n}$ be a square piece of $G_{r}^{n}$, an $n \times n$ dynamic graph. Let $p(n)$ be the probability that $\hat{G}_{r}^{n}$ contains $G_{a}^{n}$. We form a vertical pile of $s / n$ such blocks to obtain $s \times n$ such dynamic graphs as in Fig. 12. After we connect each two adjacent squares, the resulting graph is the same as $G_{r}^{n}$.

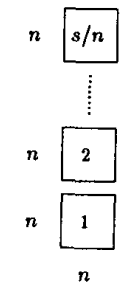

Fig. 12. Pile of $\hat{G}_{r}^{n}$ for the proof of Lemma 5.1 .

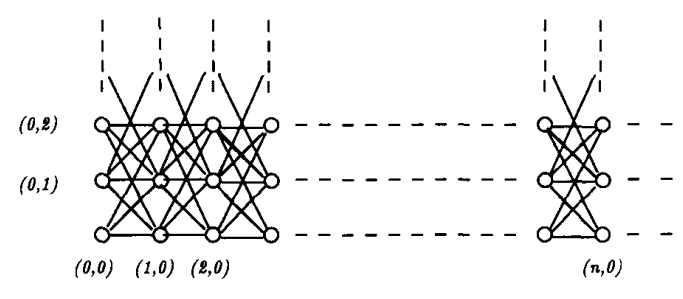

Fig. 13. Dynamic graph construction for Corollary 5.3 .

Since connections between two squares can only increase the reliability, the probability that there does not exist a working linear array in this big graph is $<(1-p(n))^{s / n}$. For any $c$, if $s>c n \log n /(-\log (1-p(n)))$, the preceding probability will be $<1 / n^{c}$. Therefore, for any reliability $\beta$, we can find a sufficiently large $s$ to achieve reliability $\beta$.

We can now prove the main result in this section.

Theorem 5.2: When $\mathcal{G}_{a}$ is a family of $d$-dimensional $\mathrm{dy}$ namic graphs, there exists an embedding architecture where $\mathcal{G}_{r}$ is a family of $(d+1)$-dimensional dynamic graphs, which can be $L R$ reliable with any given $\beta$.

Proof: As earlier, we construct a reduced graph from the given dynamic application graph $G_{a}$. The most general form of a reduced graph is a web. Thus, without loss of generality, we need only prove the theorem for the case of the application graph being a family of $d$-dimensional webs. We can use the same construction and reconfiguration method as we did in the previous lemma.

From the preceding reconfiguration method, after $k \leq$ $F T\left(G_{a}^{n}\right)$ nodes have failed, we need to change at most $2 \vec{k}$ nodes. The following corollary shows that when $d=1$, we can reduce this to exactly $k$ nodes.

Corollary 5.3: When $\mathcal{G}_{a}$ is a family of linear arrays, there exists an embedding architecture where $\mathcal{G}_{r}$ is a family of 2-D dynamic graphs with edge degree $4 m+2$, where $m$ is any constant $\geq 2$, such that after any $k \leq F T\left(G_{a}^{n}\right)$ nodes have failed, we only need to change $k$ nodes.

Proof: First construct the dynamic graph as shown in Fig. 13, where there are $s$ nodes in each column: each node $(i, j)$ connects to $(i+1, j+m),(i+1, j+m-1), \cdots,(i+$ $1, j), \cdots,(i+1, j-m+1),(i+1, j-m)$.

The reconfiguration method is the same as in Lemma 5.1. Let $F T\left(G_{a}^{n}\right)<s$ for each $G_{a}^{n}$ in the family, and allocate nodes of $G_{a}^{n}$ to different columns as earlier. The number of nodes that need to be changed after $k$ nodes in one column have failed is at most $\lceil k / m\rceil \times 2-1$. This is the worst case, so $D R(k, n)=\max (\lceil k / m\rceil \times 2-1, k)=k$, if $m \geq 2$. 
Similar constructions work for $d$ dimensions.

\section{Conclusions AND OPEn Problems}

Our main result is that it is difficult for dynamic graphs to maintain both local reconfigurability and a fixed level of reliability. More precisely, the dynamic graph must be of dimension at least one greater than the application graph to have both properties.

The problem of considering the tradeoffs among the size of redundant graphs (the number of edges), reconfigurability, and reliability needs to be studied further. A class of simple layered graphs with a logarithmic number of redundant edges is proposed in [19] which can maintain both finite reconfigurability and a fixed level of reliability for a wide class of application graphs. By sacrificing finite reconfigurability, they also construct highly reliable structures with the asymptotically optimal number of edges for one-dimensional and treelike array architectures. However, the redundant graphs resulting from the constructions are not dynamic graphs. It would be interesting to consider the construction of redundant graphs that are restricted to be dynamic graphs, which are more easily implemented than less regular graphs.

\section{REFERENCES}

[1] F. R. K. Chung, F. T. Leighton, and A. L. Rosenberg, "Diogenes: A methodology for designing fault-tolerant VLSI processing arrays," in Proc. IEEE Int. Symp. Fault-Tolerant Computing, Milano, June 1983 pp. $26-32$.

[2] P. R. Cappello and K. Steiglitz, "Digital signal processing applications of systolic algorithms," in CMU Conf. VLSI Systems and Computations, H. T. Kung, B. Sproull, and G. Steele, eds. Rockville, MD: Computer Science Press, Oct. 1981, pp. 19-21.

[3] J. W. Greene and A. E. Gamal, "Configuration of VISI arrays in the presence of defects," J. Asso. Comp. Mach, vol. 31, pp. 694-717, Oct. 1984.

[4] J. P. Hayes, "A graph model for fault-tolerant computing systems," IEEE Trans. Comput., vol. C-25, no. 9, pp. 875-884, Sept. 1976.

[5] M. J. lacoponi and S. F. McDonald, "Distributed reconfiguration and recovery in the advanced architecture on-board processor," in Proc IEEE Int. Symp. Fault-Tolerant Computing, Montreal, June 1991, pp. $436-443$.

[6] K. Iwano and K. Stciglitz, "Testing for cycles in infinite graphs with periodic structure," in Proc. 19th Anmual ACM Symp. Theory Computing, New York, May 1987, pp. 46-55.

[7] K. Iwano and K. Steiglitz, "Planarity testing of doubly periodic infinite graphs," Networks, vol. 18, no. 3, pp. 205-222, Fall 1988

[8] K. Iwano and K. Steiglitz, "A semiring on convex polygons and zerosum cycle problems," SIAM J. Computing, vol. 19, no. 5, pp. 883-901 Oct. 1990.

[9] H. T. Kung, "Why systolic architectures?" IEEE Comput., vol. 15, no. 1 , pp. $37-46$, Jan. 1982

[10] H. T. Kung and M. S. Lam, "Fault tolerant VLSI systolic arrays and two-level pipelines," J. Parall. Distr. Proc., vol. 8, pp. 32-63, 1984.

[11] S. Y. Kung, VLSI Array Processors. Englewood Cliffs, NJ: PrenticeHall, 1988
[12] S. Y. Kung, K. S. Arun, R. J. Gal-Ezer, and D. V. Bhaskar Rao, "Wavefront array processor: Languages, architecture, and applications," IEEE Trans. Comput., vol. C-31, pp. 1054-1066, Nov. 1982.

[13] S. Y. Kung, S. N. Jean, and C. W. Chang, "Fault-tolerant array processors using single track switches," IEEE Trans. Comput., vol. C-38, no. 4, pp. 501-514, Apr. 1989

[14] S. D. Kugclmass and K. Steiglitz, "A scalable architecture for lattice-gas simulation," J. Computational Physics, vol. 84, pp. 311-325, Oct. 1989.

[15] T. Leighton and C. E. Leiserson, "Wafer-scale integration of systolic arrays," IEEE Trans. Comput., vol. C-34, no. 5, pp. 448-461, 1985.

[16] J. Orlin, "Some problems on dynamic/periodic graphs," Progress in Combinatorial Optimization, W. R. Pulleyblank, ed. Orlando, FL: Academic Press, 1984, pp. 273-293.

[17] V. P. Roychowdhury, J. Bruck, and T. Kailath, "Efficient algorithms for reconfiguration in VLSI/WSI arrays," IEEE Trans. Compt. vol. C.39, no. 4 , pp. 480-489, Apr. 1990.

[18] M. Sami and R. Stcfenelli, "Reconfiguration architecture for VLSI processing arrays," in Proc. IEEE Int. Symp. Fault-Tolerant Computing, 1986, pp. 712-722

[19] E. H.-M. Sha and K. Steiglitz, "Explicit constructions for reliable reconfigurable array architectures," in Proc. 3rd IEEE Symp. Parallel Distributed Process., Dallas, TX, Dec. 1991, pp. 640-647.

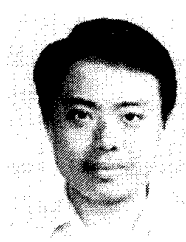

Edwin Hsing-Mean Sha received the B.S. degree in computer science from National Taiwan University, Taipei, Taiwan, in 1986, and the M.S. and Ph.D. degrees in computer science from Princeton University in 1990 and 1992 , respectively.

He is now Assistant Professor of Computer Science and Engineering at the University of Notre Dame, Notre Dame. IN. His research interests include fault tolerant computing, testing, VLSI architectures, high-level synthesis in VLSI, and algorithens.

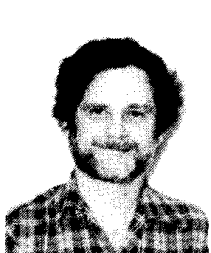

Kenneth Steiglitz (S'57-M'64-SM'79-F'81) was born in Weehawken, NJ, on January 30, 1939. He reccived the BE.E (magna cum laude), MEE. and Eng.Sc.D. degrees from New York University, New York, NY, in 1959, 1960, and 1963, respectively.

Since September 1963 he has been at Princeton University, Princeton, NJ, where he is now Professor of Computer Science, teaching and conducting research on parallel architectures, signal processing, optimization algorithms, and cellular automata. He is the author of Introduction to Discrete Systems (New York: Wiley, 1974), and coauthor, with C. H. Papadimitriou, of Combinatorial Optimization: Algorithms and Complexity (Englewood Cliffs, NJ: Prentice-Hall, 1982).

Dr. Steiglitz served two terms as member of the IEEE Signal Processing Society's Administrative Committee, as chairman of their Technical Directions Committee, member of their VLSI Committee, their Digital Signal Processing Committee, and as their awards chairman. He is an Associate Editor of the journal Networks, and is a former Associate Editor of the Journal of the Association for Computing Machinery. A member of Eta Kappa Nu, Tau Beta $\mathrm{Pi}$, and Sigma Xi, he was elected Fellow of the IEEE in 1981, received the Technical Achievement Award of the Signal Processing Society in 1981, their Society Award in 1986, and the IEEE Centennial Medal in 1984. 